\title{
Major conceptual change required to improve lung cancer: see a respiratory physician
}

\author{
J.K. Field*, C. Brambilla\#
}

In this issue of the European Respiratory Journal, FERGUSON et al. [1] have provided valuable evidence of the importance of patients with a diagnosis of lung cancer seeing a respiratory physician (RP). Their data support the concept that patients who have had the fortune of seeing an RP benefit. The authors are careful to indicate that the patients who did not see an RP were generally older and had more extensive disease. However, even after correcting for this factor, a significant advantage was observed for those who were seen by an RP. Their findings support the national guidelines and it is clear that patients seeing an RP are more likely to be managed by a multidisciplinary team and receive active treatment. However, there is no information available to associate this with a better quality of survival.

Unfortunately, in the UK there has been little change in the 5-yr survival figures for lung cancer since George VI, who died from the disease in 1952 . In the UK, $<5 \%$ of patients survive 5 yrs after diagnosis, which is a poor statistic when considered alongside many other European countries 5-yr survival rates of $\leqslant 14 \%$ [2].

There are currently four major areas that could improve lung cancer mortality: 1) smoking cessation programmes; 2) identification of individuals at risk of developing lung cancer; 3) referral and management of lung cancer patients to respiratory physicians; and 4) treatment strategies based within national and international clinical trials.

The best way to control lung cancer is to reduce cigarette smoking in the population, primarily through prevention and secondarily through smoking cessation. This has to be taken on board at national government levels with the objective of banning smoking in all public places in Europe. However, even after stopping smoking, the long-term smokers remain at a high risk of lung cancer and there is no established cancer control policy for individuals who are already at high risk due to prolonged exposure to tobacco smoke or occupational exposures. The recent developments in biological and radiological techniques have opened up the possibility of early diagnosis of lung cancer leading to improved prognosis and reduced mortality [3,4]. The development of molecular genetic early diagnostic techniques, aimed at the recognition of individuals who are at risk of developing lung cancer prior to clinical symptoms, is the way forward, using both sputum and serum-based approaches [5, 6]. However, the most promising early detection methods currently involve spiral computed tomography (CT) screening.

Lung cancer screening by low-dose spiral CT has produced promising results in several preliminary studies in the USA

\footnotetext{
* Roy Castle International Centre for Lung Cancer Research, The University of Liverpool, Liverpool, UK. \#Dept de Medecine Aigue Specialisee, Unite de Pneumologie, Grenoble, France.

Correspondence: J.K. Field, Roy Castle International Centre for Lung Cancer Research, The University of Liverpool, London Road, Liverpool L3 9TA. Fax: 44 1517948989. E-mail: J.K.Field@liv.ac.uk
}

and Japan [7, 8, 9]. This new technology has made it possible to detect minimal small $(>5 \mathrm{~mm})$ asymptomatic nodules. The prognosis for such patients may be significantly improved compared to current patients with lung cancer, even those with Stage 1 disease. The development of spiral CT scanning to detect small lung cancers is the most promising discovery in the field of early lung cancer detection in the last 20 yrs. However, randomised controlled trials on spiral CT screening must be undertaken prior to using this technology and such trials are currently being undertaken by the National Cancer Institute (NCI) and certain European groups within a European Union/USA spiral CT collaboration.

Chemotherapy-based treatment strategies for nonsmall cell lung cancer (NSCLC) have been recently reviewed by Thatcher [10] and Rintoul and SETHi [2]. However, many of these approaches provide little benefit (2-4 months) in survival when compared with "best supportive care". Recent publications in gene profiling of lung cancer $[11,12]$ illustrate the developmental path new drug targets will take. BEER et al. [11] demonstrated that histopathology is insufficient to predict disease progression and clinical outcome in lung adenocarcinoma. They showed that gene-expression profiles based on microarray analysis can be used to predict patient survival in early-stage lung adenocarcinomas. HEIGHWAY et al. [12] took a different approach to the examination of gene profiling, using a panel of complimentary deoxyribonucleic acid microarrays, comprising 47,650 transcript elements, and carrying out gene expression profiling of 39 resected primary human nonsmall cell lung tumours versus normal lung tissue. Analysis of the deregulated expression genes in this study is aiding identification of future genes that may be used in developing targeted drug therapies.

Three of the major approaches to defeating lung cancer are in the developmental stages, whereas the findings of FERGUSSON et al. [1] could be implemented immediately if the culture of referral and management of lung cancer patients was changed. There are few approaches to lung cancer that could have an immediate affect; this is the one that should be implemented now.

\section{References}

1. Fergusson RJ, Thomson CS, Brewster DH, Brown $\mathrm{PH}$, Milroy R, on behalf of the Scottish Cancer Trials Lung Group and the Scottish Cancer Therapy Network. Lung cancer: the importance of seeing a respiratory physician. Eur Respir J 2003; 21: 606-610.

2. Rintoul RC, Sethi T. The lung cancer paradox: time for action. Thorax 2002; 57: Suppl. 2, II57-II63.

3. Hirsch FR, Franklin WA, Gazdar AF, Bunn PA. Early detection of lung cancer: clinical perspectives of recent advances in biology and radiology. Clin Cancer Research 2001; 7: 5-22.

4. Field JK, Brambilla C, Hirsch FR, et al. Molecular 
biomarkers workshop: a European strategy for developing lung cancer molecular diagnostics in high risk populations. Lung Cancer 2001; 31: 339-345.

5. Ahrendt SA, Chow JT, Xu LH, et al. Molecular detection of tumor cells in bronchoalveolar lavage fluid from patients with early stage lung cancer. J Natl Cancer Inst 1999; 91: 332-339.

6. Liloglou T, Maloney P, Xinarianos G, et al. Cancer-Specific Genomic Instability (CSGI) in bronchial lavage: a molecular tool for lung cancer detection. Cancer Res 2001; 61: 1624 1628.

7. Henschke CI, McCauley DI, Yankelevitz D, et al. Early Lung Cancer Action Project: overall design and findings from baseline screening. Lancet 1999; 354: 99-105.
8. Sone S, Takashima S, Li F, et al. Mass screening for lung cancer with mobile spiral computed tomography scanner. Lancet 1998; 351: 1242-1245.

9. Ellis SM, Husband JE, Armstrong P, Hansell DM Computed tomography screening for lung cancer: back to basics. Clin Radiol 2001; 56: 691-699.

10. Thatcher N. New drugs in small cell lung cancer. Suppl Tumori 2002; 4: S26-S27.

11. Beer DG, Kardia SL, Huang CC, et al. Gene-expression profiles predict survival of patients with lung adenocarcinoma. Nat Med 2002; 8: 816-824.

12. Heighway J, Knapp T, Boyce L, et al. Expression profiling of primary non-small cell lung cancer for target identification. Oncogene 2002; 21: 7749-7763. 